\title{
Robust Invariant Sets and Active Mode Detection for Discrete-time Uncertain Descriptor Systems
}

\author{
Ye Wang ${ }^{1}$, Sorin Olaru ${ }^{2}$, Giorgio Valmorbida ${ }^{2}$, Vicenç Puig ${ }^{1}$ and Gabriela Cembrano ${ }^{1,3}$
}

\begin{abstract}
In this paper, the computation of robust invariant sets for discrete-time uncertain descriptor systems is investigated. The studied descriptor systems are assumed to be regular and stable subject to unknown but bounded disturbances. The robust invariant sets of both causal and non-causal descriptor systems are studied. Transformations for causal and non-causal descriptor systems are used in the characterization of the effect of the disturbances. For causal descriptor systems, two robust positively invariant (RPI) sets are computed separately. For non-causal descriptor systems, an RPI set and a robust negatively invariant (RNI) set can be found. As a result, the general RPI set of a descriptor system can be obtained from a linear projection image of these two sets. Besides, active mode detection method is proposed based on set invariance theory.
\end{abstract}

\section{INTRODUCTION}

Set invariance has played an essential role in control theory with a variety of applications to constrained dynamical systems, uncertain control systems as well as their related (constrained) control design [1]. It is instrumental for control strategies such as reference governor [2], fault diagnosis [3] and fault-tolerant control [4]. The robust positively invariant (RPI) set and minimal RPI (mRPI) set have been well studied and the approximation of the mRPI set has been investigated and summarized in [5]. Alternatively, ultimate bounds of dynamical systems [6] can provide RPI sets and the computation of such ultimate bounds can be of relative low complexity. An iterative strategy is presented in [4] and leads to mRPI estimation based on the computation of ultimate bounds.

In different scenarios, due to mass, volume or energy conservation laws, the differential or difference equations describing a dynamical system are coupled with a set of algebraic equations. This class of systems is called descriptor systems. Instances of such systems are water distribution networks [7], electrical circuits [8], and economic models [9]. A well-posed descriptor system, for which a solution exists and is unique, is said to be regular. Regularity, however, does not imply causality and models of interest in economy are non-causal, see e.g. the Leontief model [9].

\footnotetext{
${ }^{1}$ Y. Wang, V. Puig and G. Cembrano are with Advanced Control Systems (SAC) Research Group at Institut de Robòtica i Informàtica Industrial (IRI), CSIC-UPC, Universitat Politècnica de Catalunya-BarcelonaTech (UPC), C/. Llorens i Artigas 4-6. 08028 Barcelona, Spain. E-mail: ywang@iri.upc.edu, vicenc.puig@upc.edu, cembranodiri.upc.edu

${ }^{2}$ S. Olaru and G. Valmorbida are with Laboratoire des Signaux et Systèmes, CentraleSupélec, CNRS, Univ. Paris-Sud, Université Paris-Saclay, 3 Rue Joliot-Curie, 91192 Gif sur Yvette, France. E-mail: sorin.olarul2s.centralesupelec.fr, giorgio.valmorbidadl2s. centralesupelec.fr

${ }^{3} \mathrm{G}$. Cembrano is also with CETaqua, Water Technology Centre, Ctra. d'Esplugues 75, Cornellà de Llobregat, 08940 Barcelona, Spain.
}

The main contribution of this paper is to present a method for the computation of robust invariant (RI) sets for discretetime regular descriptor systems. For the case of non-causal descriptor systems, we decouple the set of states into causal and anti-causal states. The computation of the mRPI set of the causal states is obtained via an iterative method with ultimate bounds. On the other hand, for the anti-causal states, we introduce robust negatively invariant (RNI) sets. Then, we apply the obtained computational results for active mode detection. Finally, some numerical examples illustrate the proposed methods.

The remainder of this paper is structured as follows: In Section II, we present the problem statement and some preliminary results. In Section III, main results on the computation of mRPI sets of causal and non-causal descriptor systems are presented. In Section IV, active mode detection methods are proposed based on set invariance theory. In Section $\mathrm{V}$, numerical examples are provided to illustrate the proposed methods. In Section VI, some conclusions are drawn.

Notation: The Minkowski addition operator is denoted by $\oplus . I_{j}$ denotes the identity matrix of dimension $j$. Let $\mathcal{X}$ and $\mathcal{Y}$ be two non-empty sets, the Hausdorff distance is defined by $d_{H}(\mathcal{X}, \mathcal{Y})=\max \left\{\sup _{x \in \mathcal{X}} \inf _{y \in \mathcal{Y}} d(x, y), \sup _{y \in \mathcal{Y}} \inf _{x \in \mathcal{X}} d(x, y)\right\}$ with $d(x, y)$ the distance between two points $x$ and $y$. For $X \in \mathbb{R}^{n \times n}$, we use $\operatorname{deg}(\operatorname{det}(X(z)))$ to denote the degree of the determinant of $X$ on variable $z$ and we also use $\lambda(X)$ to denote the set of eigenvalues of matrix $X$.

\section{Problem Statement and Preliminary Results}

\section{A. Discrete-time Descriptor Systems}

Consider the discrete-time linear time invariant (LTI) descriptor system

$$
\operatorname{Ex}(k+1)=A x(k)+B_{w} w(k),
$$

where $x \in \mathbb{R}^{n}$ denotes state vector, $w \in \mathbb{R}^{q}$ denotes the disturbance vector, $A \in \mathbb{R}^{n \times n}, B_{w} \in \mathbb{R}^{n \times q}$ and $E \in \mathbb{R}^{n \times n}$ with $\operatorname{rank}(E)=r \leq n$. For a descriptor system (1), under the disturbance-free condition, we have the following properties.

Lemma 1 (Regularity, causality and asymptotic stability): The following conditions can be used to verify [9]

- Regularity: the matrix pair $(E, A)$ is regular if $\operatorname{det}(z E-$ $A)$ is not identically zero.

- Causality: the matrix pair $(E, A)$ is causal if $\operatorname{deg}(\operatorname{det}(z E-A))=\operatorname{rank}(E)$. 
- Stability: the matrix pair $(E, A)$ is asymptotically stable if $|\lambda(E, A)|<1, \forall \lambda(E, A) \in\{z \mid \operatorname{det}(z E-A)=0\}$.

The descriptor system (1) is said to be admissible if it is regular, causal and asymptotically stable. In this paper, we study the descriptor systems that are not necessarily admissible, i.e. we consider descriptor systems which involves anti-causal states.

Assumption 1: The descriptor system (1) (the matrix pair $(E, A))$ is regular and asymptotically stable.

We now establish transformations that decompose the descriptor systems in subsystems suitable for analysis.

Definition 1 (Equivalent systems): The descriptor system (1) with $\left(E, A, B_{w}\right)$ is said to be restrictively equivalent to the descriptor system with $\left(\tilde{E}, \tilde{A}, \tilde{B}_{w}\right)$ under the transformation $(P, Q)$ if there exists a pair of non-singular matrices $P \in \mathbb{R}^{n \times n}$ and $Q \in \mathbb{R}^{n \times n}$ satisfying $Q E P=\tilde{E}, Q A P=\tilde{A}$ and $Q B_{w}=\tilde{B}_{w}$.

For a given descriptor system (1), we now present two standard equivalent forms that are of interest.

1) Dynamic Decomposition Form: Consider the descriptor system (1) with $\operatorname{rank}(E)=r$, there always exists a transformation $(M, N)$ yielding

$$
M E N=\left[\begin{array}{cc}
I_{r} & 0 \\
0 & 0
\end{array}\right], M A N=\left[\begin{array}{cc}
A_{1} & A_{2} \\
A_{3} & A_{4}
\end{array}\right], M B_{w}=\left[\begin{array}{l}
B_{w 1} \\
B_{w 2}
\end{array}\right],
$$

with $A_{1} \in \mathbb{R}^{r \times r}, A_{2} \in \mathbb{R}^{r \times(n-r)}, A_{3} \in \mathbb{R}^{(n-r) \times r}, A_{4} \in$ $\mathbb{R}^{(n-r) \times(n-r)}, B_{w 1} \in \mathbb{R}^{r \times q}$ and $B_{w 2} \in \mathbb{R}^{(n-r) \times q}$.

Lemma 2 (Dynamic decomposition form [10]): The descriptor system (1) is causal if and only if there exists a transformation in the sense of Definition 1, giving (2) with a non-singular block $A_{4}$.

Lemma 3 (Equivalent causal descriptor system):

A causal descriptor system defined in (1) with $\operatorname{rank}(E)=r$ can be transformed in a dynamical form as

$$
\tilde{x}(k+1)=\tilde{A} \tilde{x}(k)+\tilde{B}_{w} \tilde{w}(k),
$$

where

$$
\begin{aligned}
\tilde{A} & =\left[\begin{array}{cc}
A_{1}-A_{2} A_{4}^{-1} A_{3} & 0 \\
-A_{4}^{-1} A_{3}\left(A_{1}-A_{2} A_{4}^{-1} A_{3}\right) & 0
\end{array}\right], \\
\tilde{B}_{w} & =\left[\begin{array}{cc}
B_{w 1}-A_{2} A_{4}^{-1} B_{w 2} & 0 \\
-A_{4}^{-1} A_{3}\left(B_{w 1}-A_{2} A_{4}^{-1} B_{w 2}\right) & -A_{4}^{-1} B_{w 2}
\end{array}\right] .
\end{aligned}
$$

and

$$
\tilde{x}(k)=N^{-1} x(k)=\left[\begin{array}{c}
\tilde{x}_{1}(k) \\
\tilde{x}_{2}(k)
\end{array}\right], \tilde{w}(k)=\left[\begin{array}{c}
w(k) \\
w(k+1)
\end{array}\right],
$$

with $\tilde{x}_{1}(k) \in \mathbb{R}^{r}$ and $\tilde{x}_{2}(k) \in \mathbb{R}^{n-r}$.

Proof: Based on the transformation $(M, N),(1)$ can be rewritten as

$$
M E N N^{-1} x(k+1)=M A N N^{-1} x(k)+M B_{w} w(k) .
$$

Using (2) and defining $\tilde{x}(k)$ as in (5), we have

$$
\begin{aligned}
\tilde{x}_{1}(k+1) & =A_{1} \tilde{x}_{1}(k)+A_{2} \tilde{x}_{2}(k)+B_{w 1} w(k), \\
0 & =A_{3} \tilde{x}_{1}(k)+A_{4} \tilde{x}_{2}(k)+B_{w 2} w(k) .
\end{aligned}
$$

Since the descriptor system is causal from Lemma 2, $A_{4}$ is invertible. Then, from (6b), we also have

$$
\tilde{x}_{2}(k)=-A_{4}^{-1} A_{3} \tilde{x}_{1}(k)-A_{4}^{-1} B_{w 2} w(k) .
$$

Substituting $\tilde{x}_{2}(k)$ in (6a) by (7), we obtain

$\tilde{x}_{1}(k+1)=\left(A_{1}-A_{2} A_{4}^{-1} A_{3}\right) \tilde{x}_{1}(k)+\left(B_{w 1}-A_{2} A_{4}^{-1} B_{w 2}\right) w(k)$.

From (7), we also have $\tilde{x}_{2}(k+1)=-A_{4}^{-1} A_{3} \tilde{x}_{1}(k+1)-$ $A_{4}^{-1} B_{w 2} w(k+1)$ then use (8) to obtain

$$
\begin{aligned}
\tilde{x}_{2}(k+1)= & -A_{4}^{-1} A_{3}\left(A_{1}-A_{2} A_{4}^{-1} A_{3}\right) \tilde{x}_{1}(k)-A_{4}^{-1} B_{w 2} w(k+1) \\
& -A_{4}^{-1} A_{3}\left(B_{w 1}-A_{2} A_{4}^{-1} B_{w 2}\right) w(k) .
\end{aligned}
$$

Hence, we obtain (3).

\section{2) Kronecker Canonical Form:}

Lemma 4 (Kronecker canonical form): The descriptor system (1) is regular if and only if there exists a transformation $(\hat{M}, \hat{N})$ yielding

$$
\hat{M} E \hat{N}=\left[\begin{array}{cc}
I_{p} & 0 \\
0 & \bar{N}
\end{array}\right], \hat{M} A \hat{N}=\left[\begin{array}{cc}
\bar{A} & 0 \\
0 & I_{(n-p)}
\end{array}\right],
$$

where $\bar{N}$ is a nilpotent matrix and $p \leq r=\operatorname{rank}(E)$.

Lemma 5 (Causality): Let the matrix pair $(E, A)$ be given in the regular form (9), then it is causal if and only if $\bar{N}=0$.

\section{B. Set Invariance}

For the regular and stable descriptor system (1), we assume the additive disturbance vector $w(k)$ to belong to a compact set $\mathcal{W}, \forall k \in \mathbb{Z}$. As a consequence of the boundedness of the disturbances and the stability, the system trajectories are expected to be confined in a set within the state space [11]. Given an initial state $x(0)$ and the unique solution of (1) denoted by $x(k), \forall k \in \mathbb{Z}$ (the time instant $k$ can be negative for the backward propagation), the following definitions are introduced in view of the set-theoretic analysis.

Definition 2 (Robust invariant set): A set $\Omega \in \mathbb{R}^{n}$ is said to be robust invariant with respect to the descriptor system (1) if $x(0) \in \Omega$ implies $x(k) \in \Omega, \forall w(k) \in \mathcal{W}$ and $\forall k \in \mathbb{Z}$.

Definition 3 (Robust positively invariant set): A set $\Omega \in$ $\mathbb{R}^{n}$ is said to be robust positively invariant with respect to (1) if $x(0) \in \Omega$ implies $x(k) \in \Omega, \forall w(k) \in \mathcal{W}$ and $\forall k \in \mathbb{Z}_{[>0]}$.

Definition 4 (Minimal RPI set): An RPI set $\Omega_{\infty} \in \mathbb{R}^{n}$ is said to be minimal RPI with respect to (1) if it is contained in every closed RPI set of (1).

Definition 5 (L-step robust negatively invariant set):

The set $\Omega \in \mathbb{R}^{n}$ is $L$-step robust negatively invariant with respect to the descriptor system (1) if the state $x(L) \in \Omega$ implies $x(L+k) \in \Omega, \forall w(k) \in \mathcal{W}$ and $\forall k \in \mathbb{Z}_{[-L, 0]}$.

\section{Robust INVARIANT SET CHARACTERIZATIONS FOR DISCRETE-TIME DESCRIPTOR SYSTEMS}

\section{A. The mRPI Set of Causal Descriptor Systems}

For a regular and stable descriptor system (1), which is also causal, the set analysis can be performed based on (3) by decomposition in two sub-spaces as in the following theorem.

Theorem 1 (mRPI set of admissible descriptor systems): Consider the admissible descriptor system (1) and two nonsingular matrices $M$ and $N=\left[\begin{array}{ll}N_{1} & N_{2}\end{array}\right]$ with $N_{1} \in \mathbb{R}^{n \times r}$ and $N_{2} \in \mathbb{R}^{n \times(n-r)}$ satisfying (2), the mRPI set $\Omega^{c}$ of the admissible descriptor system (1) is given by the 
Minkowski addition of the image of two mRPI sets for two subcomponents as

$$
\Omega^{c}=N_{1} \Phi_{1} \oplus N_{2} \Phi_{2},
$$

with

$$
\begin{aligned}
& \Phi_{1}=\bigoplus_{i=0}^{\infty} \tilde{A}_{1}^{i} \tilde{B}_{w 1} \mathcal{W}, \\
& \Phi_{2}=\left(-A_{4}^{-1} A_{3} \Phi_{1}\right) \oplus\left(-A_{4}^{-1} B_{w 2} \mathcal{W}\right) .
\end{aligned}
$$

Proof: From (4), the descriptor system (1) is equivalent to the following two subsystems

$$
\begin{aligned}
& \tilde{x}_{1}(k+1)=\tilde{A}_{1} \tilde{x}_{1}(k)+\tilde{B}_{w 1} w(k), \\
& \tilde{x}_{2}(k+1)=\tilde{A}_{2} \tilde{x}_{1}(k)+\tilde{B}_{w 2} w(k)+\tilde{B}_{w 3} w(k+1),
\end{aligned}
$$

with $\quad \tilde{A}_{1}=A_{1}-A_{2} A_{4}^{-1} A_{3}, \quad \tilde{A}_{2}=-A_{4}^{-1} A_{3}\left(A_{1}-A_{2} A_{4}^{-1} A_{3}\right)$, $\tilde{B}_{w 1}=B_{w 1}-A_{2} A_{4}^{-1} B_{w 2}, \quad \tilde{B}_{w 2}=-A_{4}^{-1} A_{3}\left(B_{w 1}-A_{2} A_{4}^{-1} B_{w 2}\right)$, $\tilde{B}_{w 3}=-A_{4}^{-1} B_{w 2}$.

Moreover, the stability implies the matrix $\tilde{A}_{1}$ is Schur. Then, the characterization of the mRPI set of $\tilde{x}_{1}$ can be obtained as in (11a) using the classical LTI notions [11]. On the other hand, the mRPI set $\Phi_{2}$ of $\tilde{x}_{2}$ can be found by a linear projection image of the set $\Phi_{1}$ in (11a), which bounds the state $\tilde{x}_{1}(k)$. This leads to

$$
\begin{aligned}
\Phi_{2} & =\tilde{A}_{2} \Phi_{1} \oplus \tilde{B}_{w 1} \mathcal{W} \oplus \tilde{B}_{w 2} \mathcal{W} \\
& =\left(-A_{4}^{-1} A_{3} \Phi_{1}\right) \oplus\left(-A_{4}^{-1} B_{w 2} \mathcal{W}\right) .
\end{aligned}
$$

By means of the Minkowski addition of the sets obtained via the images defined by the matrices $N_{1}$ and $N_{2}$, we obtain the RPI set of the admissible descriptor system (1).

Lemma 6 (Ultimate bounds [6]): Given a standard LTI system (1) with $E=I_{n}$ and the Schur matrix $A \in \mathbb{R}^{n \times n}$, the Jordan decomposition form of $A=V \Lambda V^{-1}$ and the compact disturbance set $w(k) \in \mathcal{W}=\left\{w \in \mathbb{R}^{q}|| w \mid \leq \bar{w}\right\}, \forall k \in \mathbb{N}_{+}$, the set $\Phi=\left\{x \in \mathbb{R}^{n}|| V^{-1} x\left|\leq(I-|\Lambda|)^{-1}\right| V^{-1} B_{w} \mid \bar{w}+\theta\right\}$ is RPI and attractive for all the trajectories of this system, where $\theta$ is an arbitrarily small vector with positive elements.

Corollary 1 (Approximation of the mRPI set): Consider the admissible descriptor system (1), an RPI approximation of the mRPI set (10) for the admissible descriptor system (1) is given by $\Omega_{0}^{c}=N_{1} \hat{\Phi}_{1,0} \oplus N_{2} \hat{\Phi}_{2,0}$ with

$$
\begin{aligned}
& \hat{\Phi}_{1,0}=\left\{\tilde{x}_{1} \in \mathbb{R}^{n_{\tilde{x}_{1}}}|| V_{1}^{-1} \tilde{x}_{1}\left|\leq\left(I-\left|\Lambda_{1}\right|\right)^{-1}\right| V_{1}^{-1} \tilde{B}_{w 1} \mid \bar{w}+\theta_{1}\right\}, \\
& \hat{\Phi}_{2,0}=\left(-A_{4}^{-1} A_{3} \hat{\Phi}_{1,0}\right) \oplus\left(-A_{4}^{-1} B_{w 2} \mathcal{W}\right),
\end{aligned}
$$

where $\tilde{A}_{1}=V_{1} \Lambda_{1} V_{1}^{-1}, \theta_{1}$ is an arbitrarily small vector with all positive elements. Furthermore, the sets $\Omega_{i}^{c}=N_{1} \hat{\Phi}_{1, i} \oplus N_{2} \hat{\Phi}_{2, i}$ with $i \in \mathbb{N}_{+}$can be constructed based on the recurrence

$$
\begin{aligned}
& \hat{\Phi}_{1, i}=\tilde{A}_{1} \Phi_{1, i-1} \oplus \tilde{B}_{w 1} \mathcal{W}, \\
& \hat{\Phi}_{2, i}=\left(-A_{4}^{-1} A_{3} \hat{\Phi}_{1, i}\right) \oplus\left(-A_{4}^{-1} B_{w 2} \mathcal{W}\right),
\end{aligned}
$$

and lead to RPI approximation of the mRPI set (10) with $\Omega_{0}^{c} \supseteq \Omega_{1}^{c} \supseteq \cdots \supseteq \Omega_{i}^{c} \supseteq \cdots \supseteq \Omega^{c}$ for any $\epsilon>0$, it exists an $i \epsilon$ $\mathbb{N}_{+}$such that $d_{H}\left(\Omega_{i}^{c}, \Omega^{c}\right)<\epsilon$.

Proof: By Lemma 6 , the RPI set $\Phi_{1}$ of $\tilde{x}_{1}$ from the subsystem (12a) can be approximated via a positive invariant set by ultimate bounds as $\hat{\Phi}_{1,0}$ in (13a). Therefore, the mRPI set $\hat{\Phi}_{2,0}$ for $\tilde{x}_{2}$ can be obtained through a linear image as

$$
\hat{\Phi}_{2,0}=\left(-A_{4}^{-1} A_{3} \hat{\Phi}_{1,0}\right) \oplus\left(-A_{4}^{-1} B_{w 2} \mathcal{W}\right) .
$$

Using [4] (see Algorithm 1), an approximation of the mRPI set (14) can be obtained in an iterative fashion with the $i$ step forward set propagation. Ultimately, given a constant $\epsilon$, the constrained $d_{H}\left(\Omega_{i}^{c}, \Omega^{c}\right)<\epsilon$ can be achieved for a finite index $i$ by exploiting the convergence of the sequence $\Omega_{i}^{c}$ to $\Omega^{c}$.

\section{B. The mRPI Set of Non-causal Descriptor Systems}

We now extend the results of the previous subsection to non-causal descriptor systems. If the descriptor system (1) is regular and stable but not causal, there still exists an unique solution at each sampling time instant and thus the set characterization of the dynamics can be investigated. For a regular descriptor system, the Kronecker canonical form (9) is used.

From Lemmas 4 and 5, a non-causal descriptor system can be transformed in (9) with a nilpotent matrix $\bar{N}$ satisfying $\bar{N} \neq 0$. As introduced in [9, Ch.8], it is always possible for a regular arbitrary matrix pair $(E, A)$ to find a suitable transformation $(\hat{M}, \hat{N})$ yielding (9). Computationally efficient methods exist to obtain these transformations [12].

By using Lemma 4, we set the new state variable with the partitioned form as

$$
\check{x}(k)=\hat{N}^{-1} x(k)=\left[\begin{array}{c}
\check{x}_{1}(k) \\
\check{x}_{2}(k)
\end{array}\right], \hat{M} B_{w}=\left[\begin{array}{c}
\check{B}_{w 1} \\
\check{B}_{w 2}
\end{array}\right],
$$

with $\check{x}_{1}(k) \in \mathbb{R}^{\check{n}_{1}}, \check{x}_{2}(k) \in \mathbb{R}^{\check{n}_{2}}$ and $n=\check{n}_{1}+\check{n}_{2}$.

Therefore, from (9) and (16), an equivalent system of a non-causal descriptor system (1) can be expressed as the following two subsystems

$$
\begin{aligned}
\check{x}_{1}(k+1) & =\bar{A} \check{x}_{1}(k)+\check{B}_{w 1} w(k), \\
\bar{N} \check{x}_{2}(k+1) & =\check{x}_{2}(k)+\check{B}_{w 2} w(k) .
\end{aligned}
$$

As shown in (17), the non-causal descriptor system (1) is stable if and only if the matrix $\bar{A}$ is Schur. Hence, the mRPI set of a non-causal descriptor system can be formulated in the following theorem.

Theorem 2 (mRPI set of non-causal descriptor systems): Consider the non-causal descriptor system (1) in the equivalent form (9) and its solutions $x(k), \forall k \in \mathbb{Z}_{>0}$. The mRPI set of the non-causal descriptor system (1) can be obtained by the Minkowski addition of the image of two invariant sets with $\hat{N}_{1}$ and $\hat{N}_{2}$ as

$$
\Omega^{n}=\hat{N}_{1} \Theta_{1} \oplus \hat{N}_{2} \Theta_{2},
$$

with

$$
\begin{aligned}
\Theta_{1} & =\bigoplus_{i=0}^{\infty} \bar{A}^{i} \check{B}_{w 1} \mathcal{W}, \\
\Theta_{2} & =\bigoplus_{i=0}^{s-1} \bar{N}^{i} \check{B}_{w 2} \mathcal{W},
\end{aligned}
$$

where $s>0$ is scalar satisfying $\bar{N}^{s-1} \neq 0$ and $\bar{N}^{s}=0$. Besides, $\hat{N}=\left[\begin{array}{ll}\hat{N}_{1} & \hat{N}_{2}\end{array}\right]$ for $\hat{N}$ in (16) with $\hat{N}_{1} \in \mathbb{R}^{n \times \check{n}_{1}}$ and $\hat{N}_{2} \in \mathbb{R}^{n \times \check{n}_{2}}$.

Proof: The non-causal descriptor system can be decomposed in two subsystems, where (17a) is a difference equation. Hence, the mRPI set of $\check{x}_{1}$ can be constructed as 
in (19a). On the other hand, from (17b), the anti-causal state $\check{x}_{2}(k)$ can be propagated as follows:

$$
\begin{aligned}
\check{x}_{2}(k) & =\bar{N} \check{x}_{2}(k+1)-\check{B}_{w 2} w(k), \\
\check{x}_{2}(k+1) & =\bar{N} \check{x}_{2}(k+2)-\check{B}_{w 2} w(k+1),
\end{aligned}
$$

and after $L$-step iterations, we have

$$
\check{x}_{2}(k)=\bar{N}^{L} \check{x}_{2}(k+L)-\sum_{i=0}^{L-1} \bar{N}^{i} \check{B}_{w 2} w(k+i) .
$$

Since $\bar{N}$ is a nilpotent matrix, there exists a finite index $s$ such that the following conditions holds $\bar{N}^{s-1} \neq 0$ and $\bar{N}^{s}=0$. Therefore, considering the structural relations $s \leq$ $n_{\check{x}_{2}} \leq L$, we have that each state vector $\check{x}_{2} \in \mathbb{R}^{n_{\check{x}_{2}}}$ is well defined as a solution of (17b) and (20) can be written as $\check{x}_{2}(k)=\sum_{i=0}^{s-1} \bar{N}^{i} \check{B}_{w 2} w(k+i)$.

With the compact set $\mathcal{W}$, the RNI set of $\check{x}_{2}$ can be explicitly computed as $\Theta_{2}=\bigoplus_{i=0}^{s-1} \bar{N}^{i} \check{B}_{w 2} \mathcal{W}=\check{B}_{w 2} \mathcal{W} \oplus$ $\bar{N} \check{B}_{w 2} \mathcal{W} \oplus \cdots \oplus \bar{N}^{s-1} \check{B}_{w 2} \mathcal{W}$. As a result, the mRPI set $\Omega^{n}$ of the non-causal descriptor system (1) can be obtained by the linear image and Minkowski addition of $\Theta_{1}$ and $\Theta_{2}$.

Note that Theorem 2 is built under the assumption that the solution of the system (1) is defined $\forall k \in \mathbb{Z}_{[>0]}$. Interestingly, the properties of the infinite-time trajectory lead to a positive invariance concept although the system is not causal. Theorem 2 need to be reconsidered whenever the trajectories are defined only for a finite-time interval.

Corollary 2 (Approximation of the non-causal mRPI set): As defined in Theorem 2, the mRPI set (18) of the noncausal descriptor system (1) can be approximated by $\Omega_{0}^{n}=\hat{N}_{1} \hat{\Theta}_{1,0} \oplus \hat{N}_{2} \Theta_{2}$, with

$$
\begin{aligned}
& \hat{\Theta}_{1,0}=\left\{\check{x}_{1} \in \mathbb{R}^{n_{\check{x}_{1}}}|| \bar{V}_{1}^{-1} \check{x}_{1}\left|\leq\left(I-\left|\bar{\Lambda}_{1}\right|\right)^{-1}\right| \bar{V}_{1}^{-1} \check{B}_{w 1} \mid \bar{w}+\bar{\theta}_{1}\right\} \\
& \Theta_{2}=\check{B}_{w 2} \mathcal{W} \oplus \bar{N} \check{B}_{w 2} \mathcal{W} \oplus \cdots \oplus \bar{N}^{s-1} \check{B}_{w 2} \mathcal{W}
\end{aligned}
$$

where $\bar{A}=\bar{V}_{1} \bar{\Lambda}_{1} \bar{V}_{1}^{-1}$ and $\bar{\theta}$ is an arbitrarily small vector with positive elements and $\hat{N}=\left[\begin{array}{ll}\hat{N}_{1} & \hat{N}_{2}\end{array}\right]$. Furthermore, any set $\Omega_{j}^{n}=\hat{N}_{1} \hat{\Theta}_{1, j} \oplus \hat{N}_{2} \Theta_{2}$ with $j \in \mathbb{N}_{+}$where the set $\hat{\Theta}_{1, j}=\tilde{A}_{1} \Phi_{1, j-1} \oplus \tilde{B}_{w 1} \mathcal{W}$ is also an RPI approximation of the mRPI set (18) with $\Omega_{0}^{n} \supseteq \Omega_{1}^{n} \supseteq \cdots \supseteq \Omega_{j}^{n} \supseteq \cdots \supseteq \Omega^{n}$. Moreover, for any $\epsilon>0$, it exists an $i \in \mathbb{N}_{+}$such that $d_{H}\left(\Omega_{j}^{n}, \Omega^{n}\right)<\epsilon$.

Proof: By Lemma 6 , the mRPI set $\Theta_{1}$ of $\check{x}_{1}$ can be approximated by $\Theta_{1} \subseteq \hat{\Theta}_{1}$ in (21a). As in Corollary 1, apply the iterative algorithm to obtain $\Omega_{j}^{n}$.

\section{The L-step RNI Set of Non-causal Descriptor Systems}

We consider the non-causal descriptor system (1) and focus on trajectories defined only on a finite-time window $x(k)$, $\forall k \in \mathbb{Z}_{[0, L]}$ with $L>0$. The dynamics obey the equivalent subsystems in (17) but the set-theoretic characterization need to be relaxed to consider the finite number of dynamical constraints as well as the non-causal particularities.

The difficulties are related to the presence of causal and anti-causal dynamics in (17a) and (17b). For (17a), the positive invariance will be the appropriate concept while for (17b), the negative invariance offers the suitable framework.
Theorem 3 (L-step RNI set): Consider the anti-causal subsystem (17b). The set $\Upsilon^{L}$ is $L$-step negatively invariant if

$$
\begin{aligned}
\Upsilon^{L} & \supseteq \bar{N} \Upsilon^{L} \oplus\left\{-\check{B}_{w 2} \mathcal{W}\right\} \\
& \supseteq \cdots \supseteq \bar{N}^{L} \Upsilon^{L} \bigoplus_{i=0}^{L-1}\left\{-\bar{N}^{i} \check{B}_{w 2} \mathcal{W}\right\} .
\end{aligned}
$$

Proof: $\quad$ For $\check{x}_{2}(k+L) \in \Upsilon^{L}, k \in \mathbb{Z}_{[-L, 0]}$, from (20), (22) can be obtained by the backward propagations.

Remark 1: Given two sets $\Upsilon^{L_{1}}$ and $\Upsilon^{L_{2}}$ which are $L_{1^{-}}$ and $L_{2}$-step RNI with $L_{1}>L_{2}$, we have $\Upsilon^{L_{1}} \supseteq \Upsilon^{L_{2}}$.

Remark 2: The set $\Theta_{2}$ in (19b) is a $L$-step RNI, $\forall L \geq 0$.

Remark 3: Let the set $\Theta_{2}$ constructed as in (19b). A $L$ step RNI set $\Upsilon^{L}$ for (17b) can be constructed iteratively starting from $\Upsilon^{0}=\Theta_{2}$ and using for $i \in \mathbb{Z}_{[1, L]}$ the recursive construction

$$
\Upsilon^{i}=\left\{\check{x}_{2} \in \mathcal{X}_{2} \mid \exists w \in \mathcal{W}, \bar{N} \check{x}_{2}-\check{B}_{w 2} w \in \Upsilon^{i-1}\right\}
$$

and $\mathcal{X}_{2} \subseteq \mathbb{R}^{\check{n}_{2}}$ is the physical constraint of the state $\check{x}_{2}$.

Theorem 4 (L-step RI set): Consider the non-causal descriptor system (1) in the equivalent form (9). The set

$$
\Omega^{L}=\hat{N}_{1} \Theta_{1} \oplus \hat{N}_{2} \Upsilon^{L}
$$

guarantees that $x(k) \in \Omega^{L}, \forall k \in \mathbb{Z}_{[0, L]}$ if $\check{x}_{1}(0) \in \Theta_{1}$ and $\check{x}_{2}(L) \in \Upsilon^{L}$.

Proof: $x(k)$ has two equivalent subcomponents $\check{x}_{1}(k)$ and $\check{x}_{2}(k)$. From (19a), $\Theta_{1}$ is RPI for $\check{x}_{1}(k)$. If $\check{x}_{1}(0) \in \Theta_{1}$, then it follows $\check{x}_{1}(k) \in \Theta_{1}, \forall k \in \mathbb{Z}_{[0, L]}$.

Meanwhile, $\Upsilon^{L}$ is the $L$-step RNI set for $\check{x}_{2}(k)$ as discussed in Theorem 3. If $\check{x}_{2}(L) \in \Upsilon^{L}$, then it follows $\check{x}_{2}(k) \in \Upsilon^{L}, \forall k \in \mathbb{Z}_{[0, L]}$. Then, using the Minkowski addition of the linear projection image one obtains (24).

Proposition 1: Consider the non-causal descriptor system (1) in the equivalent form (9) and define a finite-time trajectories $x(k), \forall k \in \mathbb{Z}_{[0, L]}$ with $L>0$. If $x(0) \in \Omega^{0}$ for $L>s$ with $\bar{N}^{s}=0$ and $\bar{N}^{s-1} \neq 0$, then $x(k) \in \Omega^{0}$ for $k \in \mathbb{Z}_{[0, L-s]}$ and $x(k) \in \Omega^{k-(L-s)}, \forall k \in \mathbb{Z}_{[L-s, L]}$, where $\Omega^{i}=\hat{N}_{1} \Theta_{1} \oplus \hat{N}_{2} \Upsilon^{i}$ with $\Upsilon^{i}$ in (23).

Proof: From (20), $x(k), \forall k \in \mathbb{Z}_{[0, L-s]}$ is contained in the RI set $\Omega^{0}=\Theta_{2}$ as defined in (24). On the other hand, the anti-causal states are contained in $\Upsilon^{i}$, which can be propagated by using (23) leading to the confinement of the finite time trajectories, $\forall k \in \mathbb{Z}_{[L-s, L]}$.

\section{Set-based Active Mode Detection for DESCRIPTOR SYSTEMS}

In this section, we propose two solutions to the problems of mode detection for a causal and a switched non-causal descriptor systems based on the characterization of RPI sets. In many critical infrastructures and cyber-physical systems, a fault can be interpreted as a change in the mode of operation [13]. Therefore, active mode detection can be seen an important step towards fault-tolerant control. 


\section{A. Two-mode Descriptor Systems}

Given the causal descriptor system

$$
E_{\sigma(k)} x(k+1)=A_{\sigma(k)} x(k)+D_{\sigma(k)} w(k)+B_{\sigma(k)} u(k),
$$

where $\sigma(k)$ is a constant function $\sigma: \mathbb{N} \rightarrow\{1,2\}$. The signal $u(k) \in \mathbb{R}^{m}$ denotes an input vector that can be used for set separation at time instant $k$ and $B_{\sigma(k)}$ is the associate input matrix.

Practically, the descriptor system (25) has two modes of functioning

$$
\begin{aligned}
& \text { Mode 1: } E_{1} x(k+1)=A_{1} x(k)+D_{1} w(k)+B_{1} u(k), \\
& \text { Mode 2: } E_{2} x(k+1)=A_{2} x(k)+D_{2} w(k)+B_{2} u(k) .
\end{aligned}
$$

Assumption 2: The matrix pairs $\left(E_{1}, A_{1}\right)$ and $\left(E_{2}, A_{2}\right)$ are admissible.

Active Mode Detection Problem for Causal Descriptor Systems: Considering that $\mathcal{W}$ is a compact polyhedral set containing the origin in its interior, the mRPI sets with respect to (26a) and (26b) can be approximated by applying Corollary 1 assuming $u(k)=0, \forall k \in \mathbb{N}_{+}$. Therefore, active mode detection using $u(k) \neq 0$ is summarized in the following steps.

1) Compute invariant approximations of the mRPI sets $\Omega_{1}$ and $\Omega_{2}$ with $u=0$ for $\left(E_{1}, A_{1}, D_{1}\right)$ and $\left(E_{2}, A_{2}, D_{2}\right)$.

2) Consider the nominal models of two modes

$$
\begin{aligned}
& E_{1} \bar{x}^{m_{1}}(k+1)=A_{1} \bar{x}^{m_{1}}(k)+B_{1} u(k), \\
& E_{2} \bar{x}^{m_{2}}(k+1)=A_{2} \bar{x}^{m_{2}}(k)+B_{2} u(k),
\end{aligned}
$$

and the states $x^{m_{1}}(k)$ and $x^{m_{2}}(k)$ of (26a) and (26b) satisfy the following conditions, $\forall k \in \mathbb{N}_{+}$

$$
\begin{aligned}
& x^{m_{1}}(k) \in \bar{x}^{m_{1}}(k) \oplus \Omega_{1}, \text { if } x^{m_{1}}(0) \in \Omega_{1}, \\
& x^{m_{2}}(k) \in \bar{x}^{m_{2}}(k) \oplus \Omega_{2}, \text { if } x^{m_{2}}(0) \in \Omega_{2} .
\end{aligned}
$$

3) Find a suitable $u(k), \forall k \in \mathbb{N}_{+}$such that $\left\{\bar{x}^{m_{1}}(k) \oplus \Omega_{1}\right\} \cap\left\{\bar{x}^{m_{2}}(k) \oplus \Omega_{2}\right\}=\varnothing$.

4) On-line monitoring: Consider the sequence of states $x(k)$ from (25). If for some time index $k$ it holds $x(k) \in$ $\bar{x}^{m_{1}}(k) \oplus \Omega_{1}$, then the trajectory is governed by the Mode 1. Alternatively, if $x(k) \in \bar{x}^{m_{2}}(k) \oplus \Omega_{2}$, then the system is governed by Mode 2 .

\section{B. Switched Non-causal Descriptor Systems}

As discussed in Section III-B, the mRPI set of non-causal descriptor system can be computed by a linear projection image of a RPI set of causal states and a finite-step RNI set of anti-causal states. With respect to the non-causal descriptor system, it is important to note that the RNI set of non-causal states will grow for a finite number of steps before the mode switch. Hence, this property can be used for identifying the mode change of a switch non-causal descriptor system.

The switched non-causal descriptor system (25) is redefined with $\sigma(k)=1, \forall k \in \mathbb{Z}_{\leq k_{0}}$ and $\sigma(k)=2, \forall k \in \mathbb{Z}_{>k_{0}}$ in two modes

Mode 1: $\hat{E}_{1} x(k+1)=\hat{A}_{1} x(k)+\hat{D}_{1} w(k)+\hat{B}_{1} u(k)$,

Mode 2: $\hat{E}_{2} x(k+1)=\hat{A}_{2} x(k)+\hat{D}_{2} w(k)+\hat{B}_{2} u(k)$, where $\hat{E}_{1}=\left[\begin{array}{cc}I_{n_{1}} & 0 \\ 0 & \bar{N}_{1}\end{array}\right], \hat{A}_{1}=\left[\begin{array}{cc}\bar{A}_{1} & 0 \\ 0 & I_{n_{2}}\end{array}\right], \hat{E}_{2}=\left[\begin{array}{cc}\bar{N}_{2} & 0 \\ 0 & I_{n_{1}}\end{array}\right]$, $\hat{A}_{2}=\left[\begin{array}{cc}I_{n_{2}} & 0 \\ 0 & \bar{A}_{2}\end{array}\right], \forall k \in \mathbb{Z}_{>k_{0}}, \bar{A}_{1} \in \mathbb{R}^{n_{1} \times n_{1}}$ and $\bar{A}_{2} \in$ $\mathbb{R}^{n_{2} \times n_{2}}$ are assumed to be Schur matrices, $\bar{N}_{1}$ and $\bar{N}_{2}$ are nilpotent matrices and not identically zero and the finite step of $\bar{N}_{1}$ and $\bar{N}_{2}$ are denoted as $s_{1}$ and $s_{2}$ such that $\bar{N}_{1}^{s_{1}}=0$ with $\bar{N}_{1}^{s_{1}-1} \neq 0$ and $\bar{N}_{2}^{s_{2}}=0$ with $\bar{N}_{2}^{s_{2}-1} \neq 0$.

The non-causal descriptor system switches from Mode 1 to Mode 2 at some moment $k_{0}$. The objective of the following procedure is to detect in finite time in which mode the switched non-causal system is operating for a finite number of steps before the mode switch.

Active Mode Detection Problem for Switched NonCausal Descriptor Systems: The problem is summarized in the following off-line and on-line procedures.

\section{Off-line procedure:}

1) Compute the mRI sets $\Omega_{1}^{n}$ and $\Omega_{2}^{n}$ for $\left(\hat{E}_{1}, \hat{A}_{1}, \hat{D}_{1}\right)$ and $\left(\hat{E}_{2}, \hat{A}_{2}, \hat{D}_{2}\right)$ by using Corollary 2 .

2) Compute the $L$-step RI set $\Omega_{i}^{L}$ for $\left(\hat{E}_{1}, \hat{A}_{1}, \hat{D}_{1}\right)$ with the corresponding index $s$ of the nilpotent matrix.

3) Find the signal $u(k)$ such that the nominal states $\bar{x}^{m_{1}}(k)$ and $\bar{x}^{m_{2}}(k)$ satisfy

$$
\begin{aligned}
& \left\{\bar{x}^{m_{1}}(k) \oplus \Omega_{1}^{n}\right\} \cap\left\{\bar{x}^{m_{2}}(k) \oplus \Omega_{2}^{n}\right\}=\varnothing, \\
& \left\{\bar{x}^{m_{1}}(k) \oplus \Omega_{i}^{L}\right\} \cap\left\{\bar{x}^{m_{2}}(k) \oplus \Omega_{2}^{n}\right\}=\varnothing,
\end{aligned}
$$

4) Consider the initial state $x(0) \in\left\{\bar{x}^{m_{1}}(0) \oplus \Omega_{1}^{n}\right\}$.

\section{On-line procedure:}

Consider a sequence of the states $x(k)$ of the switched non-causal descriptor system and check the following conditions:

1) If $x(k) \notin\left\{\bar{x}^{m_{1}}(k) \oplus \Omega_{1}^{n}\right\} \quad$ and $\quad x(k) \quad \in$ $\left\{\bar{x}^{m_{2}}(k) \oplus \Omega_{2}^{n}\right\}$, then the non-causal system is switched from Mode 1 to Mode 2.

2) If $x(k) \notin\left\{\bar{x}^{m_{1}}(k) \oplus \Omega_{1}^{n}\right\} \quad$ and $x(k) \quad \in$ $\left\{\bar{x}^{m_{1}}(k) \oplus \Omega_{i}^{L}\right\}$, then a switch from Mode 1 to Mode 2 will occur at most $(k+s-i)$.

3) If $x(k-1) \in\left\{\bar{x}^{m_{1}}(k) \oplus \Omega_{i}^{L}\right\}$ and $x(k) \notin$ $\left\{\bar{x}^{m_{1}}(k) \oplus \Omega_{i+1}^{L}\right\}$, then this switch from Mode 1 to Mode 2 occurs at time $k$.

\section{ILLUSTRATIVE EXAMPLES}

A. Active Mode Detection: A Two-mode Descriptor System

Given a two-mode causal descriptor system (26) with

$$
\begin{gathered}
E_{1}=E_{2}=\left[\begin{array}{lll}
1 & 0 & 0 \\
0 & 1 & 0 \\
0 & 0 & 0
\end{array}\right], D_{1}=D_{2}=\left[\begin{array}{cc}
0.5 & 0.6 \\
1.2 & 0.2 \\
0 & 0.8
\end{array}\right], \\
A_{1}=\left[\begin{array}{ccc}
0.42 & 0.95 & 0 \\
0.1 & 0.35 & 0 \\
0.5 & 0.8 & 1
\end{array}\right], A_{2}=\left[\begin{array}{ccc}
0.65 & 1.2 & 0 \\
0 & 0.01 & 0 \\
0.5 & 0.8 & 1
\end{array}\right] .
\end{gathered}
$$

From these two matrix pairs $\left(E_{1}, A_{1}\right)$ and $\left(E_{2}, A_{2}\right)$, it can be verified that both are admissible. Let

$$
w(k) \in \mathcal{W}_{3}=\left\{w \in \mathbb{R}^{2}|| w \mid \leq\left[\begin{array}{ll}
0.02 & 0.01
\end{array}\right]^{T}\right\} .
$$

Therefore, the mRPI sets of two modes can be obtained by applying Corollary 1. The computation results of two mRPI sets are shown in Fig. 1(a). An input signal and its distribution matrices are chosen as $B_{1}=B_{2}=\left[\begin{array}{lll}1 & 1 & 0\end{array}\right]^{T}$, 


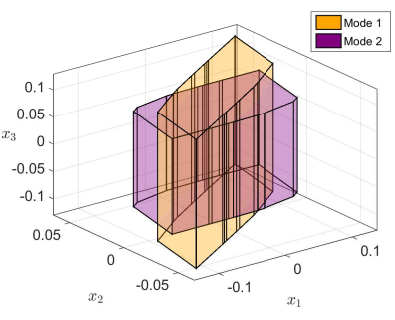

(a) The mRPI sets

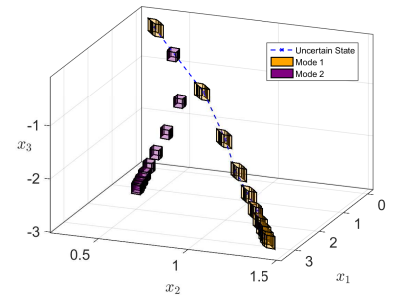

(b) Mode detection
Fig. 1. The mRPI sets and the mode detection result of the two-mode descriptor system

and $u(k)=0.5, \forall k \in \mathbb{N}_{+}$. The set separation result is shown in Fig. 1(b). This figure shows two state sets for two-mode descriptor system are separated by the selection of the input signal. Besides, the system states are propagating in the blue dashed line. From Fig. 1(b), this state is staying in the orange set. Hence, it implies that the system is in Mode 1.

\section{B. Active Mode Detection: A Switched Non-causal Descrip- tor System}

Given a switched non-causal descriptor system including two modes (29) as

$$
\begin{aligned}
& \hat{E}_{1}=\left[\begin{array}{llll}
1 & 0 & 0 & 0 \\
0 & 1 & 0 & 0 \\
0 & 0 & 0 & 0.75 \\
0 & 0 & 0 & 0
\end{array}\right], \hat{A}_{1}=\left[\begin{array}{cccc}
0.42 & 0.95 & 0 & 0 \\
0.1 & 0.35 & 0 & 0 \\
0 & 0 & 1 & 0 \\
0 & 0 & 0 & 1
\end{array}\right], \hat{D}_{1}=\left[\begin{array}{cc}
0.2 & 0.6 \\
0.9 & 0.5 \\
0.06 & 0.96 \\
1.5 & 0.15
\end{array}\right], \\
& \hat{E}_{2}=\left[\begin{array}{cccc}
0 & 0.48 & 0 & 0 \\
0 & 0 & 0 & 0 \\
0 & 0 & 1 & 0 \\
0 & 0 & 0 & 1
\end{array}\right], \hat{A}_{2}=\left[\begin{array}{cccc}
1 & 0 & 0 & 0 \\
0 & 1 & 0 & 0 \\
0 & 0 & 0.65 & 0.8 \\
0 & 0 & 0 & 0.01
\end{array}\right], \hat{D}_{2}=\left[\begin{array}{cc}
0.06 & 0.96 \\
1.5 & 0.15 \\
0.2 & 0.6 \\
0.9 & 0.5
\end{array}\right],
\end{aligned}
$$

and let

$$
\begin{aligned}
& x(k) \in \mathcal{X}=\left\{x \in \mathbb{R}^{4}|| x \mid \leq\left[\begin{array}{llll}
0.1 & 0.1 & 0.1 & 0.1
\end{array}\right]^{T}\right\}, \\
& w(k) \in \mathcal{W}_{4}=\left\{w \in \mathbb{R}^{2}|| w \mid \leq\left[\begin{array}{ll}
0.02 & 0.01
\end{array}\right]^{T}\right\} .
\end{aligned}
$$

We choose matrices $\hat{B}_{1}=\left[\begin{array}{llll}2 & 1 & 0.1 & 0.65\end{array}\right]^{T}, \hat{B}_{2}=$ $\left[\begin{array}{llll}0.1 & 0.65 & 2 & 1\end{array}\right]^{T}$, and the input signal $u(k)=0.25$, $\forall k \in \mathbb{N}_{+}$. As shown in Fig. 2, the system states of the noncausal system are switched between two modes. From $k=1$ to $k=2$, the state $x(k)$ is located in the set $\left\{\bar{x}^{m_{1}}(k) \oplus \Omega_{1}^{n}\right\}$. Hence, the switched non-causal system (25) is identified as functioning in Mode 1. At $k=3, x(k) \notin\left\{\bar{x}^{m_{1}}(k) \oplus \Omega_{1}^{n}\right\}$ and $x(k) \in\left\{\bar{x}^{m_{1}}(k) \oplus \Omega_{i}^{L}\right\}$ implies there is a switch forthcoming. And at $k=4$, the conditions $x(k-1) \in$ $\left\{\bar{x}^{m_{1}}(k) \oplus \Omega_{i}^{L}\right\}$ and $x(k) \notin\left\{\bar{x}^{m_{1}}(k) \oplus \Omega_{i+1}^{L}\right\}$ are satisfied. Hence, the switch to Mode 2 occurs at $k=4$.

\section{CONCLUSION}

Several RPI sets of causal and non-causal descriptor systems have been characterized in this paper. In particular, it is shown that the non-causal descriptor system can be transformed into two subsystems including causal and anticausal states. It is possible to find an equivalent formulation to compute the RPI sets for each subsystem. In order to compute the RPI set of non-causal descriptor systems, we have also introduced the RNI set with a finite horizon. The RPI and RNI sets can be obtained through forward

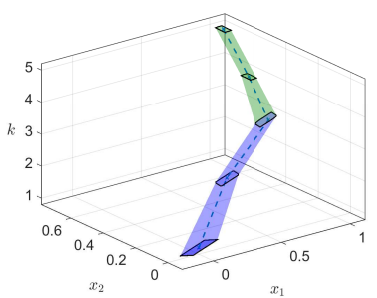

(a) $\left(x_{1}, x_{2}\right)$ space (b) $\left(x_{3}, x_{4}\right)$ space

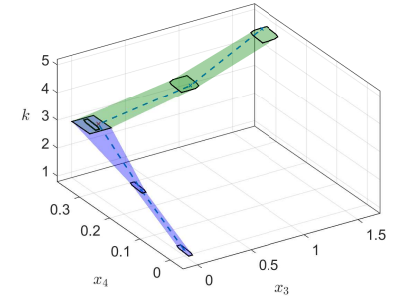

Fig. 2. The mode detection result of the switched non-causal descriptor system

and backward propagations. As an application, an active mode detection strategy has been proposed. A descriptor system with different modes can be detected and identified by implementing the RPI set separation. Future work includes the control design for the set separation in the framework of descriptor systems.

\section{ACKNOWLEDGEMENT}

This work has been partially funded by the Spanish Government and FEDER through the projects CICYT ECOCIS (ref. DPI2013-48243-C2-1-R), CICYT DEOCS (ref. DPI2016-76493-C3-3-R) and CICYT HARCRICS (ref. DPI2014-58104-R).

\section{REFERENCES}

[1] F. Blanchini, "Set invariance in control," Automatica, vol. 35, no. 11, pp. 1747 - 1767, 1999

[2] F. Stoican, S. Olaru, M. Seron, and J. D. Doná, "Reference governor design for tracking problems with fault detection guarantees," Journal of Process Control, vol. 22, no. 5, pp. 829-836, 2012.

[3] F. Xu, V. Puig, C. Ocampo-Martinez, F. Stoican, and S. Olaru, "Actuator-fault detection and isolation based on set-theoretic approaches," Journal of Process Control, vol. 24, no. 6, pp. 947-956, 2014.

[4] S. Olaru, J. D. Doná, M. Seron, and F. Stoican, "Positive invariant sets for fault tolerant multisensor control schemes," International Journal of Control, vol. 83, no. 12, pp. 2622-2640, 2010.

[5] S. Raković, E. Kerrigan, K. Kouramas, and D. Mayne, "Invariant approximations of the minimal robust positively invariant set," IEEE Transactions on Automatic Control, vol. 50, no. 3, pp. 406-410, 2005.

[6] E. Kofman, H. Haimovich, and M. Seron, "A systematic method to obtain ultimate bounds for perturbed systems," International Journal of Control, vol. 80, no. 2, pp. 167-178, 2007.

[7] Y. Wang, V. Puig, and G. Cembrano, "Non-linear economic model predictive control of water distribution networks," Journal of Process Control, vol. 56, pp. 23-34, 2017.

[8] R. Riaza, Differential-algebraic systems: Analytical aspects and circuit applications. New York, USA: World Scientific Publishing Company, 2008.

[9] L. Dai, Singular Control Systems. Berlin Heidelberg, Germany: Springer, 1989.

[10] G. Duan, Analysis and Design of Descriptor Linear Systems. New York, USA: Springer, 2010.

[11] I. Kolmanovsky and E. Gilbert, "Theory and computation of disturbance invariant sets for discrete-time linear systems," Mathematical problems in engineering, vol. 4, no. 4, pp. 317-367, 1998.

[12] A. Varga, Solving Fault Diagnosis Problems. Springer, 2017.

[13] F. Blanchini, D. Casagrande, G. Giordano, S. Miani, S. Olaru, and V. Reppa, "Active fault isolation: A duality-based approach via convex programming," SIAM Journal on Control and Optimization, vol. 55, no. 3, pp. 1619-1640, 2017. 